\title{
The Topolotron: A High Beta Device with Topological Stability
}

Keith H. Brown

H. R. P. Ferguson

J. H. Gardner

Larry V. Knight

larry_knight@byu.edu

H. Mark Nelson

Follow this and additional works at: https://scholarsarchive.byu.edu/facpub

Part of the Electrical and Computer Engineering Commons

\section{Original Publication Citation}

Brown, K. H., et al. "The Topolotron: A High Beta Device with Topological Stability." Plasma

Science, IEEE Transactions on 4.3 (1976): 162-5

\section{BYU ScholarsArchive Citation}

Brown, Keith H.; Ferguson, H. R. P.; Gardner, J. H.; Knight, Larry V.; and Nelson, H. Mark, "The Topolotron: A High Beta Device with Topological Stability" (1976). Faculty Publications. 777.

https://scholarsarchive.byu.edu/facpub/777 accepted for inclusion in Faculty Publications by an authorized administrator of BYU ScholarsArchive. For more information, please contact ellen_amatangelo@byu.edu. 
IEEE Transactions on Plasma Science, Vol.PS-4, No.3, September 1976

THE TOPOLOTRON: A HIGH BETA DEVICE WITH TOPOLOGICAL STABILITY

By

K. H. Brown*, H. R. P. Ferguson, J. H. Gardner,

L. V. Knight, and H. M. Nelson

Brigham Young University

Provo, Utah 84602

Received $7 / 30 / 75$

Abstract

The concept of topological or structural stability is introduced and its importance in the magnetic confinement of plasmas is discussed. Topological stability requires the presence of a pair of limit cycles in the magnetic field configuration. This paper deals with the design of an experimental device possessing limit cycles. The design includes a high beta $(\beta \approx 1)$, high density $\left(\sim 10^{16}\right)$, hot $(\sim 100 \mathrm{eV})$ hygrogen plasma which is to be compressed by a factor of about 5 in a toroidal device of $25 \mathrm{~cm}$ average major radius with a capacitor bank rise time of less than $2 \mu \mathrm{sec}$. Two shaped toroidal coils with opposing currents and the poloidal compression coils have been designed to give a pressure balance equilibrium and establish the limit cycles. This device could be used to determine the physical significance of topological stability in plasma confinement.

\section{Introduction}

Usually discussions of stability in plasma confinement deal with dynamic, or ordinary stability, characterized by a minimum in the potential energy. This paper will discuss another type of stability: topological stability. Ordinary or dynamic stability can be described as follows: if the magnetic bottle used for confinement is disturbed, as it will be by inevitable fluctuations in the plasma currents, the bottle is said to have ordinary stability if it remains near the original configuration, but it is unstable if the disturbance grows and permits the escape of the plasma.

Topological stability is a less familiar concept and is concerned with the topological character of the system of trajectories or phase portrait defined by the vector magnetic field on the plasma surface. If, under sufficiently small continuous changes of the parameters, the phase portrait of the vector field makes a discontinuous change into a topologically different configuration, analogous to a tearing or breaking, then the system is topologically unstable. If the small changes of the parameters do not change the topological character of the vector field, i.e., if it undergoes merely a bending or stretching, then the system is topologically stable. An instability involving a change of topology is potentially catastrophic. The study of these instabilities is often called catastrophe theory. It is possible for a system to possess ordinary stability but not topological stability or vice versa. Ideally, a system would possess both types of stability.

The application of topological stability to magnetic field configurations has been discussed elsewhere. ${ }^{1}, 2,3,4,5$ It is the purpose of this paper to present a specific experimental design which should exhibit topological stability and could be used to examine the physical significance of topological stability in plasma confinement.
Topological Systems

In a high beta $(\beta \approx 1)$ experiment, with no cusps or singularities and with axial symmetry, there are only three distinct topologies. These three cases are shown in Figure 1. In case (a) the magnetic field lines are closed, i.e., the path of any given line eventually returns to any given starting point on that line. The magnetic field has a rational rotation transform number. In case (b) the lines are open, infinitely long topological arcs. and never return to any given starting point on that line. This is referred to as an ergodic topology and has an irrational rotation transform number.

It should be pointed out that any change in direction of either system of field lines, however small, is capable of altering the topology of the lines from all closed to all open or vice versa. This is an excellent example of a system that is topologically unstable. In this case the topological instability leads to an MHD instability. The closed magnetic field lines in (a) are subject to "resonance" phenomena such as the global interchange instability. Because the ergodic field lines in (b) are not topologically stable, small perturbations can change the confining ergodic topology to a non-confining topology with closed field lines. This is analogous to shattering the magnetic bottle.

It is expected that ordinary instability can be controlled or managed by feedback stabilization. When a system suffers from topological instabilities, the changing topological nature of the vector magnetic field makes feedback stabilization of ordinary instabilities impossible. We claim, therefore, that topological stability is of primary importance in determining a viable design for thermonuclear confinement.

Except for cusped devices, present high beta toroidal plasma experiments are of the type shown in (a) and (b). The proposed experiment discussed in this paper is of the type shown in (c). This third configuration is the simplest configuration to exhibit topological stability. The magnetic field is everywhere tangent to the surface and is characterized by a finite number of closed magnetic field lines with all other magnetic field lines asymptotic to these. These closed field lines are called limit cycles and divide the surface into two regions with oppositely directed toroidal current components induced in these regions. The two closed field lines are purely toroidal. The rotational transform for this configuration is zero. While small perturbations in physical parameters can cause a change from (a) to (b) or vice versa, they cannot produce a change from (a) or (b) to (c) or vice versa, i.e., (c) is a topologically stable configuration.

Theoretical considerations indicate that the limit cycle configuration has ordinary stability against global interchanges for arbitrary aspect ratios and is stable against other MHD instabilities such as the sausage, kink, and flute modes for sufficiently large aspect ratios. ${ }^{6,7,8}$ Indeed consideration of the 
problem of topological stability leads one to believe that quite possibly plasma confinement could be significantly improved by including topological stability in experimental designs. Although topological stability may have an important role in the design of a successful magnetic confinement device, no experiment has yet been performed to explore this concept. The experiment discussed below would be the first of the (c) class and hence the first to exhibit topological stability. The experiment would have the limited goals of determining the following:

(1) Whether the limit cycle configuration, which should be topologically stable, can be created in a laboratory plasma.

(2) Whether such a limit cycle configuration has physical significance in improving plasma confinement.

\section{Computer Simulation}

A computer simulation of the plasma compression has aided in designing the experiment. ${ }^{9}, 10$ One of the problems in designing such an experiment is the complex nature of the inductive interactions between the plasma and the external coils. The computer was used to aid in determining the currents, voltages, etc., necessary to produce a plasma equilibrium with limit cycles. In the computer simulation, the plasma was regarded as a perfectly conducting gas, and its surface was represented by a set of circular loops of resistanceless wires. ${ }^{11}$ Figure 2 shows a typical computer calculation of the plasma shape during the compression with the associated poloidal magnetic field configuration. Due to the symmetry of the plasma shape and magnetic fields, only the upper half plane is shown. There is also a strong toroidal field present which is not in the figure. The two external toroidal coils in the experimental design, discussed below, are represented by wires in the computer model and are shown in Figure 2. Current directions in the plasma surface and in the toroidal coils are indicated by the circle and cross symbols. The poloidal or theta coil was included in the model exactly, without using the wire approximation, but is not shown in the figure. The computer model also included in the coil circuits the capacitors in the energy bank and external inductances representing cables, spark gaps, etc. Using familiar concepts of inductance to include the complex nature of the interaction between the plasma and the external coils, the induced currents in the various wires and the resulting forces between them were calculated and used to model the plasma compression. A snowplow model was included in the compression phase. The computer program was used to determine the optimum coil configuration, the vacuum chamber shape, information about the plasma equilibrium, and the energy bank parameters. Figure 3 shows the plasma in various stages of compression as predicted by the computer simulation. The times are indicated. At approximately $2 \mu \mathrm{sec}$, the currents in the coils were approximately at their maximum values, and the capacitors in the computer model were crowbarred.

\section{Experimental Design}

The coil design decided upon is shown in Figure 4. The vacuum vessel and plasma are also shown. An expanded view of this is shown in Figure 5 . Notice that the poloidal or theta current is in the outermost or theta coil. The two inner coils, called the I-coil and the C-coil, are toroidal and conduct current in opposite directions as shown.

It is recognized that the plasma shape shown has unfavorable curvature on the outer edge which could lead to instabilities. The computer simulation indicates that the outer toroidal field coil will provide some stabilization for the plasma. Motion of the plasma toward that coil increases the induced currents in the plasma, thus increasing the magnetic fields between the coil and the plasma. The increased magnetic pressure provides a restoring force to correct the outward plasma drift. This would be similar to the correctional force observed in the screw pinch. It was observed in the screw pinch experiment that a small outward displacement of the plasma column resulted in a counterforce on the plasma due to compression of the magnetic field between the plasma and the metal shell surrounding the torus. 12

An appropriate plasma regime for the creation of topologically stable limit cycles is the same as for the fast theta pinches since the necessary large toroidal field on the limit cycles must be created by a theta current. Also a high beta $(\beta \approx 1)$, thin skin model is postulated in the proof of topological stability. Physically this requires fast rising fields. Consequently, the proposed experiment is modeled after well-known theta pinch experiments such as the Scylla-IV series at Los Alamos.

There will be separate energy banks connected to each of the coils with a total bank energy of about $25 \mathrm{~kJ}$. The projected rise time is less than $2 \mu \mathrm{sec}$, with a peak field of about 8 kilogauss in the vacuum region. When the currents in the three coils are approximately at their maximum values, the capacitor banks will all be crowbarred. The plasma is expected to reach a temperature of about $100 \mathrm{eV}$ at a density of about $10^{16}$ particles per cubic centimeter at the maximum compression which is achieved by a fast rising shock wave driven by the three energy banks. The plasma will be preionized by an $r-f$ signal into the plasma followed by the discharge of a single $1.85 \mu \mathrm{F}$, $50 \mathrm{kV}$ capacitor through the theta coil. This discharge will be crowbarred at the maximum current to avoid the negative voltages which result during a ringing discharge. These parameters are summarized in Table I.

The shape of the plasma cross section and a mapping of the magnetic field profile would give sufficient information to establish the existence of limit cycles. This information, as well as equilibrium and stability data, can be found using standard diagnostic techniques.

\section{Summary}

An experiment has been proposed which will examine the stability properties of a high beta, toroidal plasma with limit cycles, which is expected to be topologically stable on the surface. The proposed experiment, known as the Topolotron, will be a small torus operated in the regime and using the technology of a fast theta pinch. This will provide the high beta environment favorable to the creation of limit cycles. The experiment is expected to determine whether limit cycles can be created experimentally and what effect a limit cycle structure has on plasma stability.

\section{References}

B. K. Harrison, J. H. Gardner, R. W. Bass, L. Fearnley, H. R. P. Ferguson, K. M. Larsen, and H. M. Nelson, "Topolotron: A Stochastically Stable Optimal Toroidal Magnetic We11," lst Annual IEEE International Conference on Plasma Science (1974).

2 J. H. Gardner, R. W. Bass, L. Fearnley, H. R. P. Ferguson, B. K. Harrison, K. M. Larsen, and H. M. Nelson, "The Topolotron, A Device for 
the Magnetic Confinement of Plasmas," Proc. of the Utah Academy of Sciences, Arts, and Letters, 50, part 2, 1 (1973).

3 L. Fearnley, R. W. Bass, H. R. P. Ferguson, J. H. Gardner, B. K. Harrison, K. M. Larsen, and H. M. Nelson, "Topological Global Stability of the Topolotron," Proc. of the Utah Academy of Sciences, Arts, and Letters, 50, part 2, 12 (1973).

4 B. K. Harrison, R. W. Bass, L. Fearnley, H. R. P. Ferguson, J. H. Gardner, K. M. Larsen, and H. M. Nelson, "Uniqueness of the Topolotron Design Relative to Structural Stability," Proc. of the Utah Academy of Sciences, Arts, and Letters, 50, part 2, 19 (1973).

5 H. M. Nelson, R. W. Bass, L. Fearnley, H. R. P. Ferguson, J. H. Gardner, B. K. Harrison, K. M. Larsen, D. E. Nielson, G. Cook, L. Scott, R. Wilson, and W. Young, "Physical Realizability of the Topolotron Configuration," Proc. of the Utah Academy of Sciences, Arts, and Letters, 50, part 2, 47 (1973).

6 R. W. Bass, L. Fearnley, H. R. P. Ferguson, H. J. Fletcher, J. H. Gardner, B. K. Harrison, and K. M. Larsen, "Topolotron's Unique Immunity to Interchange Instabilities," Internal Report at Brigham Young University (29 August 1975). Also submitted for publication to Physics of Fluids.

7 K. M. Larsen, "MHD Stability of a Topolotron," Internal Report at Brigham Young University (19 June 1975).

8 K. Larsen, "Kink Instabilities in a Topolotron," Bu11. Am. Phys. Soc., Series II, 20, 1311 (October 1975).

9 H. M. Nelson, K. H. Brown, and C. A. Hart, To be published.

10 H. M. Nelson, C. A. Hart, and K. H. Brown, "A Computer Sequence to Aid in the Design of a Fast Toroidal Theta Pinch Apparatus for High Beta Plasma Compression--With Application to the
Topolotron," Bu11. Am. Phys. Soc., Series II, 20, 1311 (October 1975).

11 D. C. Stevens, "Some Magnetohydrodynamic Free Boundary Equilibria," Physics of Fluids, 17, no. 1, 222 (January 1974).

12 C. Bobeldijk, L. H. Th. Rietjens, P. C. T. Van der Laan, and F. Th. DeBats, "Equilibrium and Stability of a Toroidal Screw Pinch." Plasma Physics, 9, 13 (1967).

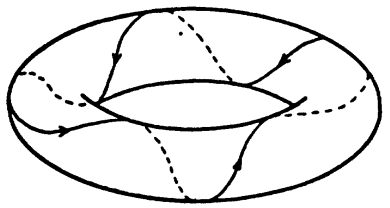

(a)



(b)

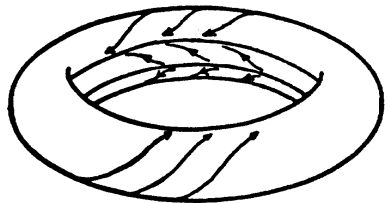

(c)

Fig. 1 The three topologies for a high beta $(\beta \approx 1)$ experiment with no cusps or singularities and axial symmetry: (a) closed field lines, (b) open field lines, and (c) limit cycle confiquration.

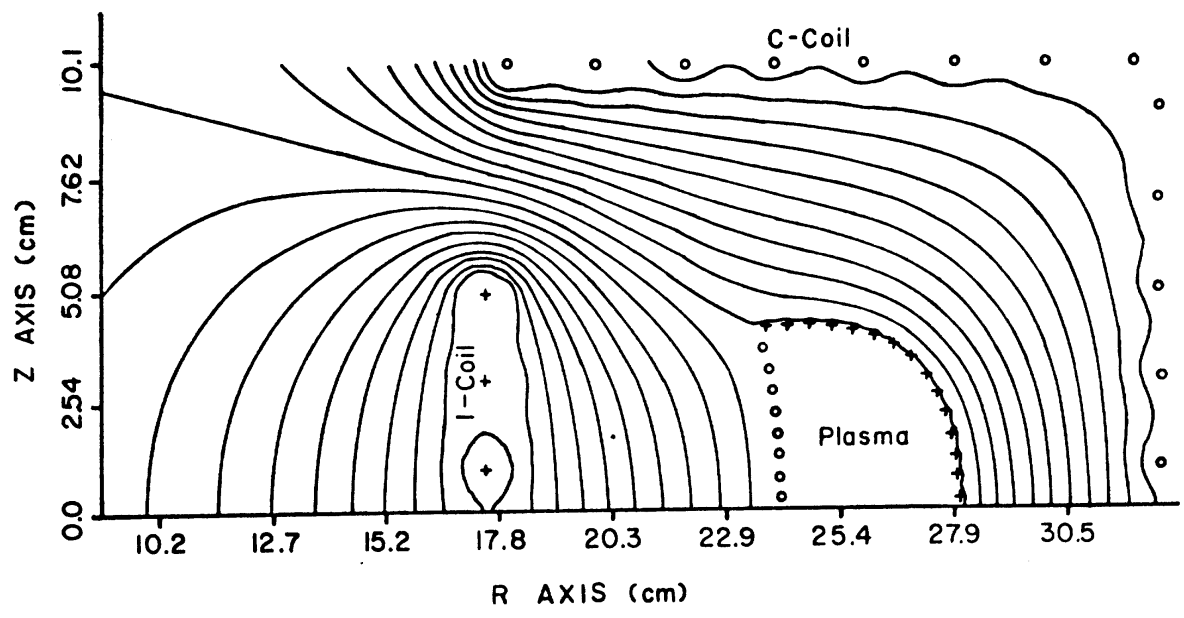

Fig. 2 Computer simulation of the plasma shape and poloidal magnetic field lines during compression. The current directions in the external coils and in the plasma surface are indicated. 




Fig. 3 Computer predicted plasma shapes during compression at times of $(a) t=0.0$,

(b) $t=0.26 \mu \mathrm{sec}$, (c) $t=0.51 \mu \mathrm{sec}$, and (d) $t=1.01 \mu \mathrm{sec}$.

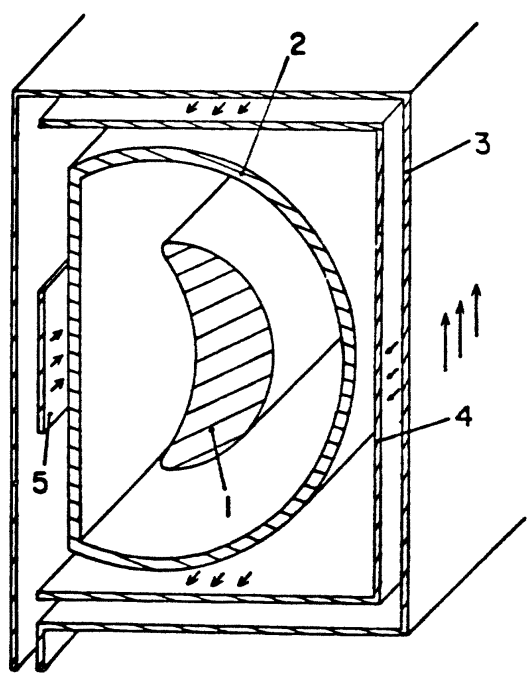

Fig. 5 Expanded view showing (1) the plasma during compression, (2) the vacuum envelope, (3) the theta coil, (4) the C-coil, and (5) the I-coil. Current directions in the field coils are indicated.

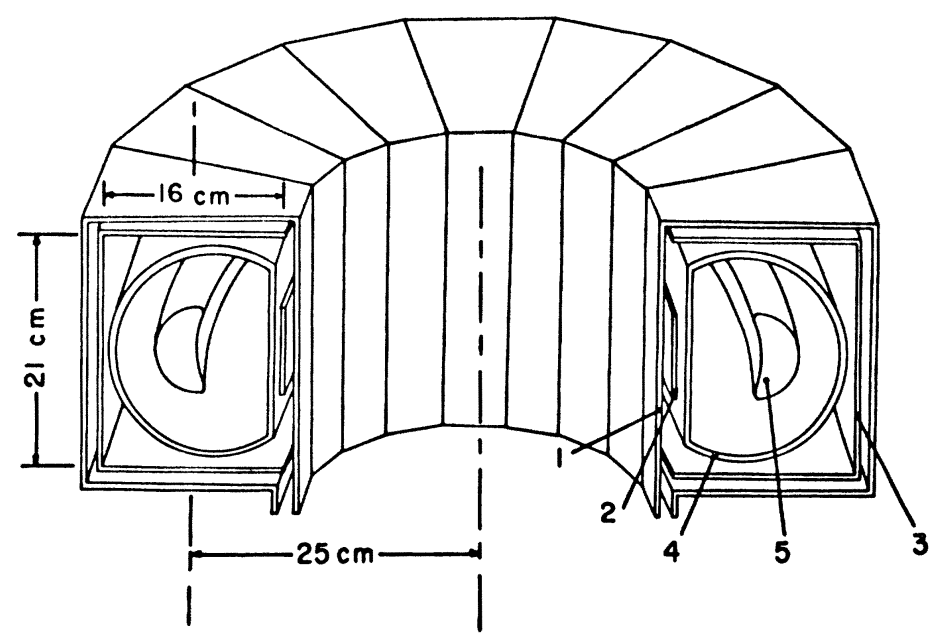

Fig. 4 Cut away view of torus showing (1) the theta or compression coil, (2) the I-coil, (3) the $\mathrm{C}$-coil, (4) the vacuum envelope, and (5) the plasma during compression. Dimensions are indicater. 